\section{IMPACTO DE UN BROTE EPIDÉMICO DE DENGUE SOBRE LAS BÚSQUEDAS EN GOOGLE EN PERÚ}

\section{[IMPACT OF A DENGUE EPIDEMIC OUTBREAK ON THE GOOGLE SEARCHS IN PERU]}

\author{
C. Hugo Arroyo-Hernández ${ }^{1,2, a}$
}

\begin{abstract}
Sr. Editor. Los problemas en salud pública tienen un impacto inmediato en medios de comunicación, en los últimos años Internet ocupa un lugar importante en ellos por la rapidez y facilidad con que se transmite la información siendo consultado especialmente por poblaciones de grandes ciudades, tanto a través de páginas especializadas como de buscadores generales ${ }^{(1,2)}$.
\end{abstract}

Estas búsquedas quedan registradas en Internet y se han utilizado con éxito para predecir el comportamiento de un brote epidémico, mostrando un potencial valor y costo efectividad como herramienta de vigilancia para identificar tempranamente brotes epidémicos, incluso antes de que este sea anunciado oficialmente por instituciones de salud; la vigilancia basada en las tendencias de búsquedas de términos específicos, ingresados por usuarios de Internet a motores de búsqueda como Google, abren además un nuevo campo de investigación denominado epidemiología de la información o infodemiología; sin embargo, esta novedosa forma de pesquisa de epidemias no ha sido diseñada para reemplazar a las redes de vigilancia tradicional ${ }^{(3-5)}$.

Con el objetivo de evaluar si existe una correlación entre las búsquedas en Google realizadas por usuarios de Internet en Perú para el término "dengue" y los casos notificados de un reciente brote en la costa norte del Perú, se elaboró un modelo con el volumen relativo de ambos, donde se incluye, además, el número de artículos periodísticos peruanos relacionados con el dengue, disponibles en Internet, buscando determinar si el incremento de búsquedas en Google es proporcional al incremento en el número de artículos periodísticos en Internet.

El número total de casos de dengue notificados por las direcciones regionales de salud de Piura, Lambayeque, La Libertad y Tumbes, se obtuvo de los boletines semanales de la Dirección General de Epidemiología

\footnotetext{
Sociedad Científica de Estudiantes de Medicina de Ica, Facultad de Medicina, Universidad Nacional San Luis Gonzaga, Ica, Perú.

2 Hospital Santa María del Socorro de Ica, Ica, Perú.

a Interno de medicina
}

Recibido: 23-08-10 Aprobado: 08-09-10
(DGE), disponibles en su página web (www.dge.gob.pe/ boletin2010.php).

La frecuencia de búsquedas realizadas por usuarios de Internet en Perú usando la palabra "dengue" se obtuvo de la aplicación para estadísticas de búsquedas de Google denominada "Google Insight" (www.google.com/insights/ search/\#), del cual se exportó los registros históricos de búsquedas durante las primeras 26 semanas del año 2010 , estos datos se expresan como volumen relativo, es decir datos normalizados en una escala del 0 al 100 , donde este último valor representa el máximo de búsquedas realizadas en el periodo de búsqueda, similar estrategia fue usada para analizar la curva de casos notificados de dengue. Los resultados se encuentran en la siguiente dirección Web: http://bit.ly/e2p5Q6

Los artículos periodísticos peruanos disponibles en Internet relacionados con el término "dengue", se contabilizaron por semanas mediante el motor de búsqueda de Google (www.google.com.pe).

Los resultados encontrados en la Figura 1, muestran un incremento en el volumen relativo de búsquedas relacionadas con el término "dengue" realizadas por usuarios de Internet, proporcional al tiempo de incremento de casos notificados de dengue en la costa norte del Perú, según las semanas epidemiológicas de la DGE.

Al evaluar la asociación entre las búsquedas en Google para dengue y los casos notificados, se obtuvo un coeficiente rho de Spearman 0,94 mostrando una correlación positiva estadísticamente significativa $p=0,001$; no se halló correlación significativa entre los casos notificados y artículos periodísticos disponibles en Internet (rho de Spearman= $-0,17, p=0,39$ ); así como entre las búsquedas en Google y artículos periodísticos disponibles en Internet (rho de Spearman $=-0,11, p=0,59$ ).

Según Google Insigth, las búsquedas en Internet relacionadas con el término "dengue" provinieron de usuarios de la ciudad de Lima, esto debido a que el volumen de búsquedas realizadas por usuarios de Internet residentes en ciudades de la costa norte del Perú no son los suficientes para ser incluidos en el logaritmo de estadísticas que esta aplicación de Google maneja.

Sin embargo, se necesitan estudios adicionales para determinar si las búsquedas en Google podrían determinar un brote epidémico indirectamente de la región donde esta ocurra. Asimismo, explorar si el incremento en el volumen de búsquedas en Google para términos relacionados con un brote epidémico ocurrido en el Perú podría comportarse como un registro de su aparición en algún periodo especifico. 


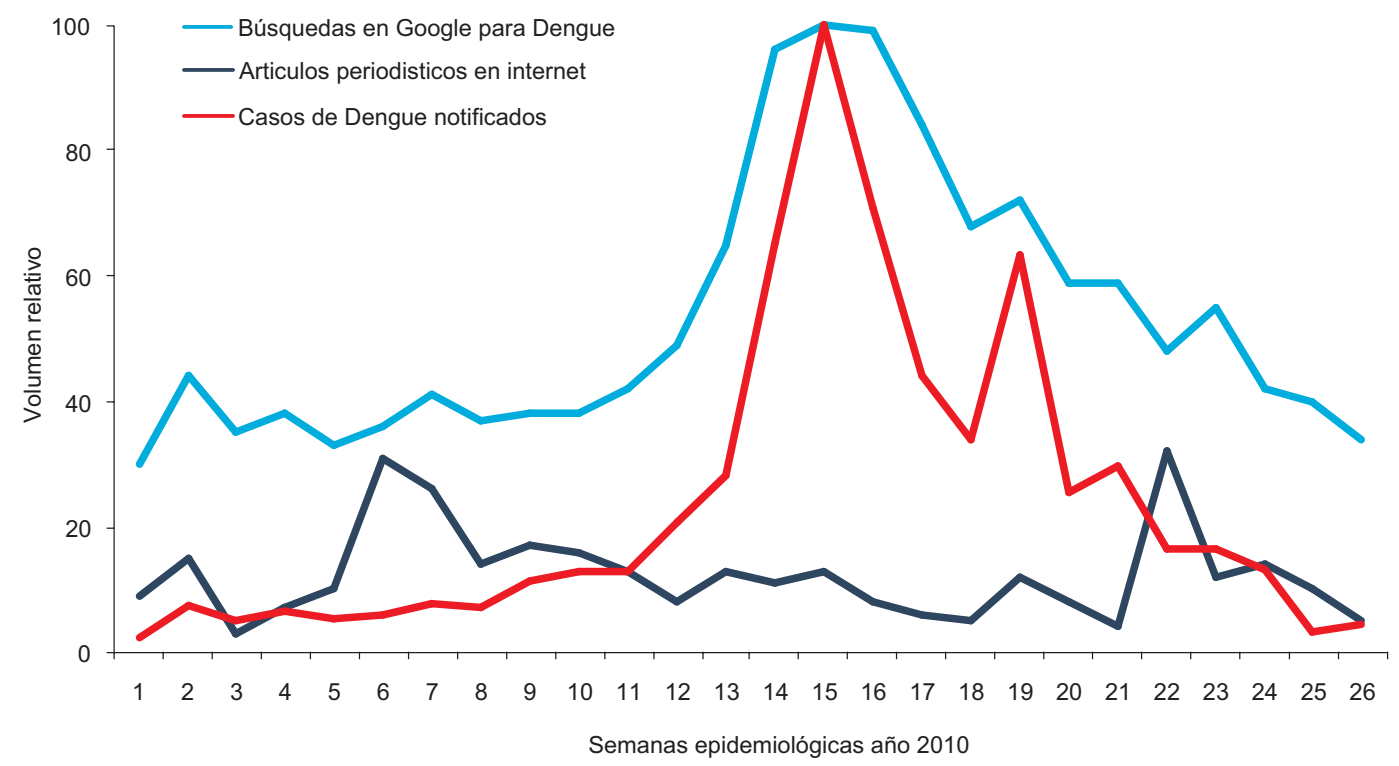

Figura 1. Curva epidémica del dengue en la costa norte del Perú, búsqueda de información en Google y notas periodísticas sobre dengue, 2010.

Una limitación de este trabajo es el problema semántico, ya que, el término "dengue" puede ser usado por usuarios de Internet para otro tipo de búsquedas no relacionadas con la enfermedad, elevando el registro con términos confusores, como se ha comunicado previamente para este tipo de estudios usando el buscador Google ${ }^{(6)}$. Durante el periodo de evaluación, encontramos que las búsquedas principales hacen referencia a los siguientes términos (los que reportaron el nivel de interés más alto): "el dengue", "dengue remix", "síntomas dengue", "el dengue remix", "dj el dengue". Este problema semántico relacionado con las búsquedas sobre salud en Google ha sido descrito previamente en la literatura ${ }^{(2,5)}$. Sin embargo, se observó que las búsquedas que experimentaron un mayor porcentaje de crecimiento durante las semanas seleccionadas con respecto a semanas anteriores fueron aquellas relacionadas con los términos "síntomas" y "tratamiento".

Si bien, los brotes epidémicos tienen además un impacto en medios de comunicación periodísticos, este no es proporcional al incremento de casos notificados, ni al incremento de búsquedas en Google durante el periodo de ocurrencia del brote; al parecer, las búsquedas en Internet para un determinado problema o emergencia sanitaria podría ser un mejor predictor que el número de notas periodísticas, lo que indicaría que el incremento de interés de los ciudadanos por un tema de salud en Internet refleja mejor la posible situación del problema, que el enfoque y atención que brindan los medios de prensa peruanos.

Esta es la primera aproximación al posible impacto que tiene un brote epidémico sobre las búsquedas en Google en un país en vías de desarrollo, actualmente estamos desarrollando modelos similares para determinar el patrón que guarda estas búsquedas en Internet sobre brotes y emergencias sanitarias en Perú.

\section{Financiamiento}

Autofinanciado.

\section{Conflictos de Interés}

El autor declara no tener conflictos de interés en la publicación de este artículo.

\section{REFERENCIAS BIBLIOGRÁFICAS}

1. Woodall J. Official versus unofficial outbreak reporting through the Internet. Int J Med Inform. 1997;47(1):31-34.

2. Nijland N, van Gemert-Pijnen J, Boer H, Steehouder MF, Seydel ER. Evaluation of internet-based technology for supporting self-care: problems encountered by patients and caregivers when using self-care applications. J Med Internet Res. 2008;10(2):e13.

3. Wilson K, Brownstein JS. Early detection of disease outbreaks using the Internet. CMAJ. 2009;180(8):829-31

4. Valdivia-Pérez A, Miguel-Benito A, Escortell-Mayor E, Monge-Corella S. Can the influenza epidemic be predicted by data from the Internet. Gac Sanit. 2010;24(1):95-96.

5. Eysenbach $\mathbf{G}$. Infodemiology and infoveillance: framework for an emerging set of public health informatics methods to analyze search, communication and publication behavior on the Internet. J Med Internet Res. 2009;11(1):e11.

6. Watts G. Google watches over flu. BMJ. 2008;337:a3076.

Correspondencia: Hugo Arroyo Hernández

Dirección: Urb. Sta. María D.- 289, distrito de Ica, Ica, Perú.

Teléfono: (051) 956936999

Correo electrónico: hugoarroyo2001@yahoo.com 